\title{
L'approche biographique et la temporalité des soins palliatifs aux enfants malades chroniques: des apprentissages qui se tissent entre la vie et la mort
}

\section{A abordagem biográfica e a temporalidade dos cuidados paliativos para crianças portadoras de doenças crônicas: aprendizados que se tecem entre a vida e a morte}

\author{
The biographical approach and the temporality of palliative care for \\ children with chronic diseases: learning that weaves between life and \\ death
}

\begin{abstract}
El enfoque biográfico y la temporalidad de los cuidados paliativos para niños con enfermedades crónicas: aprendizaje que teje entre la vida y la muerte
\end{abstract}

\begin{abstract}
Resumé: La confrontation à la mort d'un enfant, à la souffrance de sa famille, aux limites et possibilités de la médecine, aux dilemmes éthiques, au sentiment de culpabilité sont des éléments présents dans la prise en charge palliative pour les professionnels de santé qui s'engagent à travailler dans ce domaine. II s'agit de différents facteurs qui interagissent à différents moments de la prise en charge et qui contribuent pour tisser un parcours de formation composé à la fois par les expériences formelles et informelles inscrites dans le quotidien du travail.

Afin de réfléchir sur les apprentissages acquis à partir de la temporalité et de la spatialité inscrite dans ce type de soin, cet article a pour but présenter les résultats d'un travail de recherche mené dans un service de réanimation pédiatrique à Paris dont les participants ont été les professionnels de santé de l'équipe médicale et paramédicale. II s'agit d'une recherche qualitative, de base anthropologique où l'approche biographique a été mise en relation avec les observations participantes, ainsi qui a guidé de façon épistémologique et méthodologique la réalisation des entretiens biographiques non directifs avec les professionnels. Les résultats montrent qu'il y a une dynamique qui se tisse entre la temporalité de la maladie de l'enfant, l'accompagnement de la famille et la prise de décision par l'équipe biomédicale qui fait émerger un espace producteur des

1 Graduada em Psicologia pela Universidade Federal Fluminense (UFF), Pós-graduada em Saúde Coletiva pela Universidade Federal do Rio de Janeiro (UFRJ), Mestre em Ciências da Saúde pelo Instituto Fernandes Figueira (IFF/FIOCRUZ) e Doutora pelo Instituto Fernandes Figueira (IFF/FIOCRUZ). Pós-doutorado pela Université de Paris 13 (Paris Nord), França. Docente da Faculdade de Medicina de Petrópolis. E-mail: camila.aloisioalves@gmail.com
\end{abstract}


apprentissages. Dans cet espace s'inscrit des apprentissages autour de la dynamique du travail en équipe, du dialogue, de la réflexion qui favorisent le développement et la consolidation d'une approche éthique autour du soin palliatif.

Mots clés: Soin palliatif. Apprentissage. Expérience, Biographisation.

Resumo: A confrontação à morte de uma criança, ao sofrimento da sua família, aos limites e possibilidades da medicina, aos dilemas éticos, ao sentimento de culpa são elementos presentes no cuidado paliativo para os profissionais de saúde que se engajam neste campo. Tratam-se de múltiplos fatores que interagem em diferentes momentos do cuidado e contribuem para tecer uma trajetória de formação composta tanto por experiências formais, quanto informais inscritas no quotidiano do trabalho.

Com vistas a refletir sobre os aprendizados adquiridos a partir das dimensões de espaço e tempo inscritos nesse tipo de cuidado, o presente artigo tempo como objetivo apresentar os resultados de um trabalho de pesquisa realizado com os profissionais de saúde em um serviço de cuidados intensivos em Paris/ França. Trata-se de uma pesquisa qualitativa, de base antropológica e biográfica, na qual foram conjugadas observações participantes com entrevistas biográficas não diretivas com os profissionais. Os resultados mostram que há uma dinâmica que se constrói entre a temporalidade da doença na criança, o acompanhamento da família e as tomadas de decisões pela equipe biomédica que contribui para erigir um espaço produtor de aprendizagens. Neste espaço inscrevem-se aprendizados em torno da dinâmica do trabalho em equipe, do dialogo, da reflexão que favorecem o desenvolvimento e a consolidação de uma abordagem ética do cuidado paliativo.

Palavras-chave: Cuidado paliativo. Aprendizado. Experiência. Biografização.

Abstract: Confrontation with the death of a child, the suffering of his family, the limits and possibilities of medicine, the ethical dilemmas, the feeling of guilt are elements present in palliative care for health professionals who engage in this field. These are multiple factors that interact at different times of care and contribute to weave a training path composed of both formal and informal experiences inscribed in the daily work. In order to reflect on the lessons learned from the dimensions of space and time inscribed in this type of care, this article aims to present the results of a research work carried out with health professionals in an intensive care service in Paris. / France. This is a qualitative, anthropological and biographical research, in which participant observations were combined with non-directive biographical interviews with professionals. The results show that there is a dynamic that is built between the temporality of the disease in children, family monitoring and decision making by the biomedical team that contributes to erect a learning space. In this space we learn about the dynamics of teamwork, dialogue, reflection that favor the development and consolidation of an ethical approach to palliative care.

Keywords: Palliative care. Learning. Experience. Biographization.

Resumen: La confrontación con la muerte de un niño, el sufrimiento de su familia, los límites y las posibilidades de la medicina, los dilemas éticos, el sentimiento de culpa son elementos presentes en los cuidados paliativos para los profesionales de la salud que participan en este campo. Estos son múltiples factores que interactúan en diferentes momentos de la atención y contribuyen a tejer un camino de capacitación compuesto por experiencias formales e informales inscritas en el trabajo diario.

Con el fin de reflexionar sobre las lecciones aprendidas de las dimensiones de espacio y tiempo inscritas en este tipo de atención, este artículo tiene como objetivo presentar los resultados de un trabajo de investigación llevado a cabo con profesionales de la salud en un servicio de cuidados intensivos en París. / Francia. Esta es una investigación cualitativa, antropológica y biográfica, en la que las observaciones de los participantes se combinaron con entrevistas biográficas no directivas con profesionales. Los resultados muestran que existe una dinámica que se construye entre la temporalidad de la enfermedad en los niños, el monitoreo familiar y la toma de decisiones por parte del equipo biomédico que contribuye a erigir un espacio de aprendizaje. En este espacio aprendemos sobre la dinámica del trabajo en equipo, el diálogo, la reflexión que favorecen el desarrollo y la consolidación de un enfoque ético de los cuidados paliativos.

Palabras clave: Cuidados paliativos. Aprendizaje. Experiencia. Biografía 


\section{INTRODUCTION}

Les maladies chroniques chez les enfants se montrent une réalité délicate, une expérience particulière de l'enfant et de sa famille qui déclenche un ensemble de changements dans la vie de l'enfant et de leur entourage. Du côté de l'enfant, la maladie chronique peut entraîner des changements dans leur développement et dans leur processus de socialisation, de scolarisation, dans leur rapport avec la famille et les autres enfants. Chez les adolescents, la chronicité d'une maladie, comme le cancer ou la détérioration d'une mucoviscidose, engage un processus d'éloignement du groupe de pairs, de transformations physiques, ce qui peut engendrer des situations de rejet et de stigmatisation (REMEDI et. al., 2009).

Du côté des familles, parmi les nombreux défis auxquels la famille se confronte dans le quotidien, il y a, par exemple, l'accompagnent de l'enfant lors des examens, des rendez-vous chez les professionnels et des moments d'hospitalisation (VIEIRA; LIMA, 2002). Faire face à la maladie chronique d'un enfant peut entraîner des facteurs de stress liés, par exemple, à la peur que l'état de santé de l'enfant se dégrade, à la gestion de la vie de famille, à la vie de couple et à la vie productive au travail (GUERINI et. al., 2012). Il s'agit donc d'une expérience qui est vécue à la fois de façon intime par l'enfant et collective compte tenu des réverbérations qui touchent la famille.

Au cours de la vie d'un enfant ou d'un adolescent maladie chronique, l'idée de la finitude de la vie devient une présence plus fréquente, ce qui s'oppose à toutes les attentes autour de cette période de la vie, où le développement et la croissance sont les éléments mis en avant. La présence de la mort dans le quotidien de la prise en charge hospitalière, soit face à la sévérité de la condition clinique de l'enfant, soit face au décès d'autre enfant hospitalisé au même moment renvoie à des sentiments de peur et d'angoisse chez la famille et chez l'enfant.

En ce qui concerne les professionnels qui s'engagent à travailler dans ce domaine, un de leurs rôles concerne aider l'enfant et sa famille à mieux vivre l'évolution de la maladie, en essayant d'éliminer la douleur physique et d'atténuer la souffrance psychologique, émotionnelle et spirituelle. La maladie chronique se montre une expérience qui n'est pas susceptible d'être stabilisée dans une temporalité prévue en amont par rapport à l'évolution de la maladie, ce qui fait que le travail de déchiffrer la douleur chronique soit essentiel (BASZANGER, 1991), ainsi que la réflexion éthique autour des décisions liées au projet thérapeutique proposé à l'enfant.

Vivre avec une maladie chronique depuis le bas âge concerne à un processus qui a différents temporalités: du malade et son adaptation face aux changements produits par la 
maladie, de la famille qui l'accompagne et des professionnels face aux étapes de diagnostic, d'exécution et évolution du projet thérapeutique. Tout évolue en permanence et au cours de cette évolution il y a une conjugaison inévitable de la temporalité de chaque individu concerné.

Prendre en charge les enfants malades chronique devient donc une expérience difficile pour les professionnels, ce qui demande des adaptations régulières dans la quête pour ériger une approche qui se construit entre le modèle de formation et les besoins des patients. Formés pour trouver la guérison et aider les malades à échapper la mort, la chronicité amène les professionnels à passer d'un rapport de guérison construit sur l'idée de sauver vers un rapport de l'accompagnement dans le soin (ALVES, 2016).

Cependant, les progrès obtenus par les sciences et les découvertes de nouvelles technologies, qui retardent le moment de la mort, ont comme conséquence une fascination par le prolongement de la vie et un camouflage de la mort dans les scénarios hospitaliers spécifiques, comme les services de réanimation et les unités de soins continus et intensifs (SILVA; KRUSE, 2013). L'obstination thérapeutique devient donc un élément à veiller face à un soin qui se matérialise lors d'une phase de la vie où les attentes se tournent vers la croissance et le développement des malades (MENEZES, 2003; MENEZES; BARBOSA, 2013). C'est-à-dire, l'obstination thérapeutique est un élément utilisé, parfois, comme excuse pour que le temps de vie se prolonge à titre de donner une survie aux enfants.

La médicalisation de la mort, dans ce contexte, est facilement prise comme une réponse fondée sur le rassemblement de conditions technologiques, techniques et scientifiques qui peuvent soutenir les professionnels de santé face à leurs attentes concernant la survie de l'enfant, et dans certains cas, de la famille aussi face à l'angoisse de perdre son enfant. Cette médicalisation devient un médiateur entre les professionnels, les malades et leurs familles, ce qui peut à la fois produire une diminution de la capacité d'écoute de vrais besoins des sujets concernés et renforcer certains tabous sur la mort dans la société (CHAVES et. al., 2011).

En opposition à l'obstination thérapeutique, les soins palliatifs se caractérisent par un soin qui vise l'amélioration de la qualité de vie de l'enfant à travers le soulagement de la douleur et d'autres signes physiques, le support aux nécessités et attentes spirituelles et psychosociales de l'enfant et de sa famille (OMS, 2003). Les soins palliatifs sont, avant tout, un abordage complet de l'être humain qui permet d'élargir la conception de la vie au-delà du diagnostic d'une maladie sans guérison et des limitations thérapeutiques, pharmacologiques et interventionnistes disponibles. 
Il est nécessaire de considérer les soins palliatifs pédiatriques en tant qu'un chemin de possibilités qui invite les professionnels à construire un nouveau sens à la maladie chronique, à la mort des enfants et qui repositionne le poids et le rôle des technologies dans le cadre de la prise en charge afin de l'enrichir de valeurs éthiques (VARGAS et. al., 2013).

Bien que les initiatives d'incorporer davantage les soins palliatifs à la prise en charge de l'enfant malade chronique soient en cours depuis quelques années, l'étude de Garros (2003) montre que, face à une maladie chronique et à sa finitude dans des services de soins continus, il y a des craintes concernant à la fois l'utilisation d'opioïdes pour contrôler la douleur et l'inconfort, et le registre écrit sur le dossier médical de ce qui s'est passé avec le malade après l'administration de ces ressources. Dans cette même perspective, Souza et. al. (2013) ont observé, dans son étude auprès des infirmiers dans une unité d'oncologie, que les professionnels manifestent le besoin d'un soutien juridique, ainsi que d'autonomie dans la promotion de la mort digne à l'enfant, ce qui configure un dilemme entre suivre une telle conduite et la réponse légale à ses actions. Ces observations contribuent à souligner les difficultés rencontrées par les professionnels en ce qui concerne les soins apportés à la fin de la vie des malades, ce qui interfère dans la compréhension et l'incorporation des soins palliatifs.

II est possible donc de comprendre que prendre la décision d'incorporer les soins palliatifs aux enfants et adolescents signifie entrer dans un domaine de conflits et de crises diverses face à la finitude de la vie de sujets si jeunes et aux dilemmes éthiques auxquels les professionnels sont confrontés. L'agir des professionnels face aux soins palliatifs demande la construction d'une base théorique et éthico-personnelle, d'un support interpersonnel et d'une intégration entre les connaissances issues de différentes sciences et savoirs. L'intention de partager les connaissances entre les différents acteurs de la prise en charge permet d'établir une voie de solidarité et d'humanisation. Le but est de donner du confort, de promouvoir du soulagement au patient (RABELLO; RODRIGUES, 2010; MORITZ et. al., 2011) et de produire une intégration entre les différents éléments de la prise en charge.

De cette manière, les soins palliatifs sont considérés comme des moyens par lesquels doivent transiter la technique, la sensibilité, l'écoute, le dialogue, la coresponsabilité tout en respectant la temporalité de chaque individu dans la compréhension de l'évolution de la maladie qui s'inscrit dans l'espace de l'hôpital avec toutes ces représentations autour de la vie et la mort.

Toutefois, agir sur cette base demande un apprentissage qui se tisse à la fois par des formations qui amènent les professionnels à comprendre la construction et la mise en place des soins palliatifs, et par des expériences informelles vécues au cœur du processus de 
travail, où les espaces de partage et d'échange existants dans les services peuvent devenir des lieux de transformations des pratiques et de construction interdisciplinaire de l'agir professionnel. II est sur cette dimension informelle de l'apprentissage que la suite de l'article se concentre.

\section{CADRE MÉTHODOLOGIQUE}

Avant de présenter les résultats concernant les apprentissages des professionnels face à la prise en charge palliative, il est important d'expliciter le cadre méthodologique de la recherche dont le but a été de comprendre la construction du parcours biographique des professionnels de santé qui travaillent en réanimation et soins palliatifs, ainsi que les apprentissages faits au cours des expériences vécues.

Cette recherche a été développée selon une approche qualitative, de base anthropologique qui a mis en dialogue la démarche de la recherche biographique en éducation et l'ethnographie. Le choix du prisme de la recherche biographique en éducation se justifie au regard de la compréhension qu'elle apporte au processus de genèse sociale des individus, afin de montrer comment ils procèdent pour signifier les événements de leur existence vécus dans l'espace social (DELORY-MOMBERGER, 2012, 2014). La narration des expériences vécues permet d'observer comment les individus inscrivent leur existence dans l'espace et le temps à partir de certains schémas discursifs, figures de langage et de syntaxe qui prennent forme au travers des mots (DELORY-MOMBERGER, 2014).

Selon Josso (2007), la narration des expériences vécues permet d'établir des liens entre le contexte social, ses transformations et la vie des individus construite à partir d'un ensemble de facteurs, comme l'héritage, les événements vécus, les relations interpersonnelles, les projets d'avenir, etc. L'identité d'un individu concerne un processus permanent d'identification et différenciation qui se forge à partir des expériences vécues. La narration permet de mettre en évidence la pluralité, la fragilité et la mobilité de ce processus de construction identitaire au cours d'un parcours de vie (JOSSO, 2007)

L'ethnographie est une approche originaire de l'anthropologie qui, de manière intégrative, rend compte de diverses dimensions de l'être humain dans la société (LAPLANTINE, 2003). La préoccupation d'une étude de base ethnographique porte sur la définition de comment un ethos d'un groupe se construit à travers les pratiques, les codes et représentations sociales. Pour Laplantine (2003), le terrain de recherche constitue le corps de l'ethnographie, où il est possible de rencontrer les individus, d'observer les pratiques et relations quotidiennes. 
À partir de ces deux approches - de la recherche biographique en éducation et ethnographique - le travail de recherche a mis en dialogue la dimension individuelle de constitution des individus dans l'espace social, professionnel et les pratiques de soins construites et partagées. Les sujets qui ont participé à la recherche sont les professionnels de santé qui composent l'équipe du service de réanimation pédiatrique d'un hôpital de référence aux enfants malades chroniques à Paris, France.

Le travail de recherche a démarré à travers l'observation participante qui permet au chercheur de prendre en compte et de saisir les activités et les relations construites par le groupe d'individus observés (LAPLANTINE, 2003). La période consacrée à l'observation participante s'est organisée autour de l'accompagnement à la fois des différents professionnels dans leurs activités quotidiennes afin de saisir les spécificités de chaque métier et des activités collectives, comme les staffs éthiques, les transmissions de l'équipe de médecins et de l'équipe d'infirmiers, le staff médico-psy de caractère interdisciplinaire, d'entre autres. Ainsi, il a été possible de comprendre l'organisation du service et la dynamique du processus de travail, ce qui permet au chercheur de saisir comment l'éthos du groupe se construit au travers des interactions sociales. Un journal de bord a été utilisé comme outil pour enregistrer les observations des expériences vécues.

À la fin du premier mois d'observation participante, les entretiens biographiques non directifs ont été démarrés auprès des professionnels. A partir de la question de démarrage "Racontez-moi comment votre parcours a commencé dans ce domaine de la réanimation et auprès des enfants malades chroniques?", les participants ont été invités à rentrer en dialogue avec le chercheur autour des événements vécus, les choix faits, les décisions prises, les situations et expériences marquantes au cours de leur parcours de formation et de pratique dans le domaine de la santé, de la réanimation et du soin palliatif. Les entretiens ont eu lieu dans un lieu approprié, soit dans le bureau des médecins, soit dans la salle des entretiens dédiée aux parents, ce qui a garanti la confidentialité et l'anonymat.

Une fois que la recherche vise comprendre la construction du parcours biographique des professionnels à partir des expériences vécues, le critère pour inviter les professionnels à participer à cette étape de la recherche a été celui du temps de travail dans le service (avoir au moins deux ans, sauf pour les internes qui restent seulement six mois. Pour cela, l'entretien a eu lieu à la fin de la période de leur internat). Un premier groupe de huit professionnels a participé avant les congés d'été (août 2018) et les autres ont participé entre les mois de septembre à décembre 2018, selon leur disponibilité. Au total, 19 entretiens ont été menés : trois infirmières, une aide soignante, une auxiliaire de puériculture, huit médecins, deux internes de médecine, deux psychologues, une assistante sociale et une psychomo- 
tricienne. Les infirmiers ont été le groupe le moins disponible pour les entretiens à cause de leur organisation institutionnelle en rotation deux mois de nuit, deux mois de jour (12x36h). Parfois, ils sont surchargés à cause du manque d'effectif et des contraintes au niveau de la gestion institutionnelle. Ces facteurs expliquent le phénomène de turn over vécu par ce groupe et observé dans le service.

L'étape d'analyse et d'interprétation s'est divisée dans deux grandes phases organisées autour des méthodes employées dans la recherche. En ce qui concerne les observations participantes, une première lecture flottante des registres du journal de bord a été faite et puis une deuxième lecture a permis de faire un travail d'analyse de contenu (BARDIN, 2016) afin de trouver les catégories principales qui ont émergé du contexte observé: processus de travail et organisation du soin.

En ce qui concerne les entretiens, l'interprétation s'est divisée en trois grandes étapes. La première consacrée à la retranscription de chaque entretien, puis l'envoi du matériel à chaque participant pour la lecture, les commentaires et les ajouts, s'ils souhaitaient. Certains professionnels ont fait des retours écrits, d'autres ont fait de commentaires lors de rencontres dans le service et d'autres n'ont pas fait de retour. La deuxième étape s'est organisée autour de l'interprétation de chaque récit, individuellement, selon la méthode herméneutique développée par Delory-Momberger en s'appuyant sur le modèle de Walter Heinz (DELORY-MOMBERGER, 2014). Ce travail a démarré avec deux moments de lecture, ce qui a permis de repérer la manière dont chacun a construit son histoire à travers les différentes formes de discours, l'emploi des mots pour signifier, souligner, illustrer et donner des reliefs plus précis aux événements passés, les motifs récurrents dans le récit, les schémas d'action construits et qui montrent comme chaque professionnel de santé a articulé les principaux thèmes et arguments évoqués dans le récit par la mise en mot de son processus de construction biographique.

De plus, les concepts de transaction (action qui produit une relation entre deux éléments), d'événements (action exercée par l'environnement sur l'être) (PINEAU ET MARIE-MICHELLE, 2012) et turning point (moment de virage dans le parcours de vie, de basculement) (ABBOUT, 2009) ont permis de saisir le travail de réflexion et raisonnement autour des expériences vécues que leur ont permis de se diriger vers la spécialité dans le domaine de formation, de faire certains choix dans la construction de sa carrière et d'avancer dans leur engagement professionnel.

Dans sa globalité, ce matériel a apporté différents résultats. Au niveau de la dimension des individus, il est possible de comprendre comment chacun s'est construit à partir des expériences vécues et des apprentissages acquis. Au niveau de la dimension collective, le regard transversal vers l'ensemble de récits a permis de saisir des points de conver- 
gence et intersection entre les différents parcours. II est ce dernier niveau qui sera mis en avant dans la suite de l'article.

\section{QUAND L'ESPACE APPORTE LES RESSOURCES POUR FAIRE CONJUGUER LES TEMPORALITÉS AUTOUR DE LA PRISE EN CHARGE}

Pour aborder les dimensions de l'espace et temps, il faut, d'abord, expliciter l'organisation de l'espace de la réanimation. II s'agit d'un service qui compte sur 32 lits divisés dans un secteur de soin continu et trois secteurs dédiés à la réanimation où les patients sont placés selon leur degré de risque/ sévérité clinique. C'est un univers clos, protégé par des barrières physiques (portes, badges, interphones et fenêtres bien fermées) afin d'empêcher et de limiter la propagation de virus et bactéries aux malades et professionnels. Divisé par des secteurs qui indiquent la sévérité des malades face à la dépendance des machines et ressources pour garantir la survie, dans cet espace clos les patients ont leur propre chambre où l'accompagnant peut s'installer sur une chaise. Chaque chambre dans le secteur de la réanimation a les ressources pour faire marcher ce corps, comme les machines de circulation extra corporelle, le respirateur, le contrôle cardiaque, d'entre autres.

Dans le secteur du soin continu, les malades sont moins branchés dans les machines et moins dépendants de cela, parce qu'ils sont soit en train de sortir de l'hospitalisation, soit en soin palliatif où la dépendance des machines devient, parfois, une obstination thérapeutique. Dans certains cas, les soins palliatifs peuvent se faire aussi dans le secteur de la réanimation. En tout cas, la permanence ou le changement de place dans les secteurs participent de forme centrale à la matérialité de l'expérience de la maladie. Comme souligne Delory-Momberger (2010, p. 51), "l'espace est constitutif de l'expérience dans la mesure où il donne à l'expérience des orientations et des contenus, qui sont d'ordre à la fois matériel et idéel". L'espace contribue donc pour faire évoluer une compréhension de la maladie au niveau matériel, social et culturel par toutes les personnes concernées.

Comme espace et temps ne doivent pas être considérés comme étant l'un à côté de l'autre (DELORY-MOMBERGER, 2010), dans ce service de réanimation pédiatrique et soin continu, la compréhension de la maladie, des codes et normes sociales et de l'évolution par laquelle les malades passent face au pronostic institue aussi une dynamique temporelle qui demande un grand effort de compréhension pour les professionnels. La première question qui se pose est donc: Pourquoi cet effort s'impose?

Pour la répondre, il faut considérer que chaque acteur de la prise en charge vit et voit le vécu de la maladie chronique chez un enfant à partir de son insertion et engagement. Les 
médecins, ancrés sur les décisions thérapeutiques, essayent de conjuguer le savoir médical à disposition avec le bien-être du patient. Savoir le moment où la prise en charge devient déraisonnable est un exercice unique à chaque cas, qui prend des contours à chaque situation et qui est dépendant du dialogue avec la famille et l'enfant. II n'y a pas une recette applicable à tous. Pour les infirmiers et auxiliaires, les plus proches des malades et leurs familles dans le quotidien du travail, la charge de travail physique et émotionnelle est énorme et, parfois, l'insistance sur le maintient en vie d'un enfant hospitalisé signifie une double violence: pour eux en tant qu'exécutants de gestes spécifiques dans la prise en charge et pour les enfants qui subissent les interventions cliniques.

Pour la famille, c'est son enfant, un membre pour qui il faut se battre. Pour un enfant, soit il ne connait pas autre réalité que celle du vivre avec la maladie et de subir la douleur, soit, quand ils sont plus grands, son positionnement suit celui de la famille. Ainsi, confrontés à la chronicité d'une maladie, chacun fabrique son expérience de l'hospitalisation à partir de ses représentations, ce qui fait multiplier le sens de l'expérience et diversifier la façon dont chacun affronte les épreuves qui traversent les soins palliatifs et la réanimation.

Ces éléments expliquent l'émergence des temporalités qui traversent les espaces institutionnels et qui donnent un rythme à la prise en charge. Bien que les situations d'urgence soient les déclencheurs des interventions bien précises, le soin du maintien en vie quotidien et de la prise en charge palliative se construisent au fur et à mesure que ces temporalités trouvent leur dynamique dans l'espace.

Cette dynamique est aussi productrice de conflits entre parents, enfants et professionnels, notamment face à la direction que le soin prend dans l'espace et temps du service. Afin d'illustrer ce propos, je met en avant un cas vécu dans le service lors de la période de l'observation participante. II s'agit d'un garçon à l'âge de 10 ans qui a fait une fausse route lors d'un repas. Sa situation a évolué vers la mort cérébrale et il se maintenait en vie branché aux appareils pour respirer. Avant d'arriver à ce niveau-là, la situation a été largement discutée en équipe, dans différents staffs éthiques où les professionnels ont constaté son irréversibilité face aux ressources que la médecine pourrait proposer. Ce jeune garçon ne reviendrait plus. Par contre, pour la famille et ses croyances, son enfant allait encore se réveiller. Pratiquants d'une religion africaine, ils ont mis en place dans sa chambre à l'hôpital, toute une sorte de rites pour guérir son enfant. De plus, dans sa tribu, le prénom de cet enfant signifiait " combattant ". Cette confrontation entre l'évaluation des médecins et le rapport de la famille à la situation de son enfant produisait des espaces de tension, surtout parce que cette famille ne maîtrisait pas bien le français et les professionnels avaient besoin d'un interprète. Pour les infirmiers et auxiliaires, la confrontation avec les pratiques parentales ajoutait du poids à une prise en charge déjà lourde face à la souffrance 
de la famille et l'état clinique de l'enfant. Pour ces professionnels, les médecins, détenteurs de la décision finale, devraient insister davantage auprès des parents pour l'extubation. Cependant, les médecins voyaient la difficulté de dialogue pour trouver un point d'intersection entre leur point de vue et celui de la famille, compte tenu la temporalité de son acceptation de la situation dans laquelle son enfant se trouvait.

La situation s'est poursuite jusqu'au point où la famille a fini pour se confronter au manque d'efficacité de ses pratiques et sa temporalité à eux a pu se rapprocher de celles des professionnels. Pendant ce temps d' "ajustement de temporalities", le sens donné au soin de cet enfant par la famille et par les professionnels a trouvé, au fur et à mesure, des points d'intersection au regard du dialogue mis en place avec la famille, sous l'aide de l'interprète, et entre les professionnels, dans les espaces de discussion dans le service. Selon un des médecins chef du service:

\footnotetext{
"...c'était très important pour nous pour se questionner sur le sens, parce que c'était difficile de se dire que ventiler cet enfant, avec un pronostics abominable, pendant 3 mois, avait du sens à part du point de vue des supérieurs de l'enfant. II n'y avait aucun sens, mais arriver... Si tu dis " non, ça n'a pas de sens", mais on nous demande de le faire, donc on le fait... tu ne peux pas travailler comme ça. II faut que ça ait du sens et c'est là que la médiation culturelle a aidé à donner du sens et pourquoi [...] on a eu, je trouve, le plus bel éclairage à la fin, c'est-à-dire, que d'un père au départ très agressif et complètement opposé à la médiation de traitement, on a fini par un décès dans les bras du papa, extubé sans ré-intubation avec une acceptation de le laisser partir et le fait qu'il décède apaisé, avec les parents apaisés en le laissant partir, donne du sens aux trois mois qui sont écoulés... " (Médecin réanimateur, 19/09/2018).
}

À travers ce cas, il est possible de comprendre comment l'espace du service est constitutif de l'expérience dans la mesure où la convivialité des acteurs les amène au dialogue, ainsi qu'il offre des moyens de conjuguer les temporalités inscrites dans l'expérience.

Cependant, une deuxième question émerge de ces réflexions : si c'est dans cet espace clos où les temporalités gagnent forme et se rendent visibles au travers des dilemmes face à l'évolution de la maladie chez l'enfant, comment l'espace peut-il servir de ressource à l'action et à la pensée sans rester restreint aux conflits entre les individus ?

Pour répondre à cette question, j'évoquerai la question posée par Christine Delory-Momberger (2010, p. 25) - "Y a-t-il une vie sans récit ?" - dans son ouvrage "La condition biographique: essais sur le récit de soi dans la modernité avancée".

Si la réponse passe par la compréhension que la vie gagne forme au travers des mots qu'on choisi pour raconter notre histoire, les expériences vécues dans ce service et qui traversent les professionnels sont-elles porteuses d'une narrative individuelle et collective. 
Ainsi, les espaces institués pour mettre en dialogue les professionnels, comme le staff éthique, deviennent des lieux d'expression narrative et de construction d'un récit collectif entre les professionnels. Le staff éthique a été crée depuis des années afin de maintenir une démarche éthique en réanimation et soin palliatif où tous les professionnels de l'équipe médicale et paramédicale du service participent, notamment ceux qui s'occupent de l'enfant cible de la discussion à chaque fois. Dans ce staff les spécialistes d'autres services sont aussi invités à participer et il devient un lieu privilégié de transformation pour les professionnels où ils peuvent faire évoluer et fabriquer de nouvelles compréhensions de la maladie et du soin.

La diversité d'éléments et d'événements présents dans les cas suivis par le service trouve donc un espace où il est possible de construire une configuration collective de la prise en charge au travers d'une mise en intrigue de l'expérience partagée par chaque professionnel. L'espace du service qui pourrait garder ses barrières et blocages à la communication en fonction des contraintes institutionnelles, s'ouvre dans le cadre de ce staff éthique et devient donc un espace de partage et apprentissage.

Pour un médecin, le staff éthique permet de se dire, de se rend compte et de mettre en accord sur la suite du projet thérapeutique "...il y a beaucoup de staff éthique où on discute de la prise en charge des malades en disant "bah, là on va trop loin, ça va être une prise en charge palliative, enfin...". (Médecin réanimateur, 09/11/2018). Selon l'assistante sociale, le staff éthique, tel qu'il est fait dans le service, devient un espace de partage de parole et de construction interdisciplinaire de la prise en charge. Dans son récit, elle mis en avant le cas d'une petite fille qui avait un problème cardiaque qui s'est compliqué d'un problème ORL. De plus, elle venait d'un pays de l'Afrique et à un moment donné, sa maman a eu des problèmes pour rester dans le territoire français à cause de son visa. Cette assistante sociale a mis en avant que cet espace de discussion éthique dans le service a permis de trouver une solution qui a pris en compte les conditions de vie de la famille: "je pense ce staff éthique et ce service ont une façon humaine de prendre en charge la globalité. En fait, alors évidemment, ce qui va primer ce sont les soins, mais pas n'importe quel prix quelque part" (Assistante sociale, 28/06/2018).

Pour une infirmière, la démarche éthique et l'implication de l'équipe dans la prise en charge sont des facteurs déterminants pour s'investir dans le domaine et dans le travail quotidien:

"...ça a joué énormément. Ça nous met, en tout cas, dans une place en tant qu'infirmière vraiment valorisante et aussi qu'ils respectent... enfin... quand on nous explique les choses, je pense que c'est facile, c'est plus facile de faire les choses quand elles sont expliquées, quand elles sont comprises et quand on avance tous ensemble plu- 
tôt que des médecins d'un côté qui disent et puis nous qui faisons. ... c'est l'esprit du service aussi de pouvoir discuter tous ensemble, de donner son avis et essayer. C'est très riche ça. C'est valorisant, mais c'est très riche." (Infirmière, 18/09/2018)

Pour une auxiliaire de puériculture, ces moments de partage permettent de comprendre les points de vue des autres et au fur et à mesure elle a pu résinifier sa pratique entre la réanimation et les soins palliatifs "...je pense que, voilà, la réanimation va réanimer. Mais non, en fait, pour moi, la réanimation c'est accompagner un patient quelque soit l'issu, mais c'est accompagner, en fait, jusqu'où il doit aller, en fait' (Auxiliaire de puériculture, 18/09/2018).

La démarche éthique établie par le service et qui gagne une forme au travers des staffs, permet donc de mettre en place une opération de configuration narrative de l'équipe, de médiatiser le rapport avec l'enfant et sa famille et de produire un sens collectif de la prise en charge. Dans cet espace institutionnel, les temporalités de la compréhension du soin et de signification de la pratique de chacun se reconfigurent.

En traçant un parallèle avec le concept de territorialisation et déterritorialisation de Guatarri (1992), l'espace de travail des professionnels est en mouvement permanent de configuration et reconfiguration face aux expériences vécues et aux questionnements et connaissances qu'elles apportent aux professionnels. Le choix institutionnel de remplir cet espace d'une démarche éthique permet de créer un champ fertile d'apprentissage hétérobiographique à partir du partage des narratives autour du soin, ce qui contribue pour augmenter le pouvoir d'agir des professionnels et pour élargir et transformer le sens qui chacun donne à son travail.

\section{QUAND LA MORT ET LA TEMPORALITÉ DES SOINS PALLIATIFS FONT ÉLAR- GIR LE SENS DE LA RÉANIMATION PÉDIATRIQUE}

Si pour la majorité des individus dans les sociétés la mort est un sujet tabou, refoulée à l'inconscient et toujours écartée des discussions dans la vie quotidienne, pour les professionnels de santé qui travaillent dans un service de réanimation pédiatrique et soin intensif la confrontation quotidienne à la mort rappelle la condition finie du parcours de tous les individus en tant qu'êtres incarnés. Tel que nous apprend Kübler-Ross (1985, p. 31), "la mort fait partie intégrante de l'existence humaine. C'est pourquoi elle a toujours été pour chacun de nous un profond sujet d'inquiétude".

Bien que le service ait des espaces de discussion et que la démarche éthique adoptée soit une source de construction à la fois interdisciplinaire de la prise en charge et hetérogobiographique de la posture et attitude des professionnels, la confrontation à la mort de l'enfant engendre de la souffrance chez les professionnels et rend lourd le 
travail quotidien, tel que leurs récits montrent: "Le début était très difficile pour moi, les 6 premiers mois... ouf... j'ai pris cher..." (infirmière, 14/06/19) ; "En fait, je pense que je ne m'attendais pas à m'en prendre autant dans la figure. J'avais vu beaucoup de décès, bien sûr, malheureusement, mais moins régulièrement..." (interne de médecine, 17/10/2018); "C'est une période sombre [l'arrivée dans le service]. Je me suis enfermé dans le travail. Une manière extrêmement coûteuse, je crois, je ne garde pas de bons souvenirs [...] C'est moi ma propre réaction par rapport à cette agression, cette violence inouïe que j'ai vécu comme une grosse violence" (médecin réanimateur, 29/06/2018) ; "La définition de réanimation c'est que tu mets en place une machine pour aider un corps qui va mourir. Si tu ne mets pas la machine, le corps va mourir. Donc, de fait, elle est traumatisante" (médecin réanimateur, 03/07/2018).

De plus, en étant dans un service qui a pour but de sauver (réanimer) les enfants et de mettre en place tout un ensemble de machines et ressources qui deviennent indispensables à sa survie, les soins palliatifs semblent être, dans un premier temps, le contrepoint de la mission institutionnelle:

...alors, effectivement quand je dis 'moi, je fais de la réa' c'est plus dans l'optique d'être utile et de se dire 'voilà, il ne va pas bien du tout, on va arriver à sortir du truc, il va... il va... il va aller mieux, il va sortir de la réa' (médecin réanimateur, 19/11/2018).

"C'est très valorisant quoi, pour le médecinc'est pour ça que j'en fait" (Médecin réanimateur, 09/11/2018).

Les professionnels qui cherchent à travailler en réanimation pédiatrique sont imprégnés de l'envie de sauver l'enfant, d'être utile pour lui redonner les moyens de continuer à vivre: "...dès que ce n'est plus de l'aigu, je commence à être un peu mal à l'aise" (médecin réanimateur, 19/11/2018)

Toutefois, dans un hôpital qui est référence pour les enfants malades chroniques, l'arrivée et la permanence de situations qui demandent un temps plus grand d'hospitalisation et une articulation entre différents professionnels au regard des impacts de la maladie sur le corps et sur la dimension de la vie de famille, font émerger un type de soin palliatif qui rentre en dialogue avec le soin de réanimation. Dans cet espace spécifique, le professionnel est amené à la fois à vivre des situations où son geste a été décisif pour la vie de l'enfant et à réfléchir si l'insistance sur certains gestes ne va pas l'amener à rentrer dans une optique déraisonnable pour un enfant en condition de fin de vie, tel que le récit d'une auxiliaire de puériculture montre: "on est beaucoup sur la réanimation, mais qu'en réanimation, on ne réanime pas toujours..." (Auxiliaire de puériculture, 18/09/2018). 
Cette ambigüité qui vient à se greffer dans l'agir des professionnels devient un élément central dans la confrontation entre les attentes construites autour du métier et la réalité imposée par le travail quotidien. Selon une infirmière, son arrivée au service a été marquée par des idées préconçues qui ont vite été remises en question: "J'étais dans tous mes espèces de valeur super héros, en disant on est infirmier, on est là pour soigner, pour sauver, etc. Tout ça a cassé la gueule en quelques jours, quoi" (Infirmière, 14/06/2018).

Ce processus de confrontation à la réalité de la réanimation et du soin continu d'un côté montre les limites de la pratique professionnelle, malgré toutes les ressources existantes pour sauver l'enfant, et de l'autre côté devient une source d'interrogation du sens donné à cette pratique. L'ensemble de situations vécues, d'événements marquants et l'espace construit au sein du service pour discuter et réfléchir autour de la prise en charge, des projets de soin des enfants et des difficultés au travail collaborent pour amener les professionnels à comprendre que le travail qui se construit dans cet univers qui conjugue réanimation et soin palliatif forge un mode d'accompagner les enfants et leurs familles, soit vers la vie, soit vers le décès. Selon une auxiliaire de puériculture "...Je pense que, voilà, la réanimation va réanimer. Mais non, en fait, pour moi, la réanimation c'est accompagner un patient quel que soit l'issu, mais c'est accompagner, en fait, jusqu'où il doit aller, en fait" (Auxiliaire de puériculture, 18/09/2018). Sa pensée rejoint celle de ses collègues qui, après être passés par la phase de confrontation aux épreuves vécues dans le service et de se socialiser à la façon de construire la prise en charge autour d'une approche éthique et du travail en équipe, se rendent compte de l'apprentissage nécessaire autour de l'accompagnement de l'enfant et de sa famille:

"Je pense que... on peut être infirmière de soin palliatif sans être allé en réanima-
tion, mais je pense que ce n'est pas possible de faire de la réanimation sans être au
clair sur les soins palliatifs. Ça, je pense, qu'il faut absolument que les infirmières en
réanimation sachent qu'on fait des soins palliatifs et que... on a cette double cas-
quette..." (Infirmière, 09/12/2018).
"...Ce que j'aime bien, c'est les soins intensifs et la réa, et pour bien le faire, je
considère qu'il y a une part de soins palliatifs dedans [...] en réanimation je me suis
retrouvée, parfois, à faire des trucs... faire des toilettes mortuaires, d'aller faire des
biopsies post mortem, des trucs un peu... et côtoyer la mort de très, très près et
apprendre à considérer que ça fait partie de notre métier aussi. Je suis contente
d'avoir appris une certaine manière d'accompagner les gens, d'essayer de faire des
soins moins lourds et tout ça..." (Médecin réanimateur, 19/11/2018).

En prenant en compte les lignes directrices de l'OMS ${ }^{2}$ dans lesquelles le soin palliatif commence dès le moment du diagnostic et se poursuit au cours de toute la vie de l'enfant

2 Disponible sur http://www.who.int/cancer/palliative/es/. Accédé le 20/09/2019 
malade, la prise de conscience des professionnels concernant la part du palliatif dans le soin en réanimation permet d'ouvrir un espace où l'accompagnement peut se mettre en $œ u v r e ~ e t$, ainsi, favoriser le développement d'une posture interdisciplinaire dans la prise en charge. Cette posture va répercuter non seulement dans la qualité de l'accompagnement proposé aux enfants et leurs familles, mais aussi dans la façon de concevoir son rôle de professionnel de santé et de voir autrement le sens de l'agir quotidien :

" le soin palliatif c'est l'accompagnement, après ça aboutit à un décès ou pas mais je ne me sens pas impuissante, parce que, en fait, tu accompagnes les parents, tu accompagnes l'enfant et, en fait, c'est justement, moi, je trouve que c'est un des moments où on se sent utile, mais de manière simple, en fait ". (Interne de médecine, 17/10/2018).

Cependant, il ne faut pas oublier que la dimension de l'espace où la prise en charge se développe doit compter sur des dispositifs qui permettent la mise en forme de l'expérience et le partage du vécu pour que les échanges désamorcent les incompréhensions, diminuent les hiatus qui peuvent émerger d'expériences qui sont extrêmement lourdes sous le plan émotionnel. La mise en commun du vécu des professionnels par l'horizontalité inscrite dans les staffs éthiques, par exemple, favorise la construction d'un sens partagé du soin, comme met en exergue un interne de médecine: "Chaque situation est unique, mais je trouve que, justement dans ce service, comme on fait des staffs très régulièrement, au final on comprend bien la prise en charge" (Interne de médecine, 17/10/2018).

Le récit d'un jeune médecin réanimateur permet de donner une visibilité à la place où les professionnels de ce service se situent et le type de posture apprise au cours des expériences vécues:

"...ll y a effectivement ceux que tu ne peux pas sauver, qui vont aller mal et qui vont décéder, parfois des maladies chroniques hyper lentes, et donc là c'est... Je pense que là, du coup, tu axes plus sur essayer de faire les meilleurs choix ... enfin, tu vois de quoi il a besoin, sa qualité de vie, ce qui est raisonnable ou pas, enfin, c'est complètement autre chose. C'est euh... essayer de prendre la meilleure décision pour l'enfant et de l'accompagner au mieux et c'est surtout pour la famille aussi, je pense. C'est une implication aussi beaucoup pour les parents, la famille et puis.... et puis dans la communication avec les, les, les personnels soignants aussi pour qu'ils tiennent le coup, parce que parfois ça dure très longtemps, tu vois ? Mais, sinon, c'est... c'est différent, mais, en fait, ça fait partie aussi de notre boulot de réanimateur" (Médecin réanimateur, 09/11/2018).

En reprenant les catégories utilisées pour interpréter les récits des professionnels, il a été possible de voir que les expériences vécues au niveau du soin palliatif ont été prises en tant qu'événements marquants au cours de la prise en charge. Elles peuvent être ainsi vues compte tenu de la mise en avant dans les récits des dilemmes éthiques autour du 
projet de soin de l'enfant, des répercussions dans l'équipe face à la lourdeur émotionnelle inscrite dans les relations avec les collègues, l'enfant et sa famille. Cependant, le fait d'avoir des espaces institutionnels pour en discuter, d'avoir un mode de fonctionnement d'équipe horizontal qui favorise le dialogue et le partage, permet aux professionnels de transformer le vécu en apprentissage dans la mesure où ils trouvent des espaces et du temps pour la construction de la mise en commun du sens donné au soin palliatif.

Ainsi, le soin palliatif devient un facteur qui s'intègre à la prise en charge en réanimation et l'élargi dans la mesure où les professionnels sont jalonnés à la fois par l'urgence et la vigilance permanente des conditions aiguës de la réanimation et le besoin d'être en mesure de prendre de bonnes décisions pour la vie de l'enfant et de l'accompagner au mieux. L'expérience de travailler avec une approche palliative dans cet univers dévoile donc d'autres formes de redonner la vie au-delà du sens lié à la technologie et aux ressources biomédicales.

\section{CONCLUSION}

La mort, sujet tabou dans notre société, objet rejeté par les professionnels de santé, antithèse de leur pratique, peut-elle être un élément d'apprentissage pour un collectif confronté au soin palliatif?

La réponse est positive si le collectif transforme l'espace dans un lieu où les représentations sociales autour de la mort gagnent visibilité et le vécu peut s'exprimer pour que les divergences deviennent un matériel d'appréciation du point de vue de l'autre afin de faire élargir la compréhension globale du vécu qui traverse à tous.

Temps et espace sont à la fois des catégories analytiques qui aident à comprendre les phénomènes vécus par les soignants et éléments qui donnent forme au processus de formation. La dynamique qui se tisse entre la temporalité de la maladie de l'enfant, du vécu de la famille et de la prise de décision par l'équipe biomédicale fait émerger un espace potentiel d'apprentissages. Cet espace rempli par une démarche éthique permet de croiser les temporalités qui traversent et organisent la compréhension des professionnels dans une dynamique d'équipe, de dialogue, de réflexion qui favorise le développement et la consolidation de l'approche en soin palliatif. 


\section{RÉFÉRENCES}

ABBOUT A. A propos du concept de " Turning Point ". In : Michel Grossetti et al., Bifurcations, La Découverte " Recherches ", 2009, p. 187-211.

ALVES, C. A. Tessituras do Cuidado as condições crônicas de saúde na infância e adolescência. Rio de Janeiro, Edotra Fiocruz, 2016.

BARDIN, L. L'analyse de contenu. Paris: Presses Universitaires de France (puf), 3ème tirage, 2016.

BASZANGER, I. Déchiffrer la douleur chronique: deux figures de la pratique médicale. Sciences Sociales et Santé, 1991, v. 9, n. 20, p. 31-78.

CANESQUI, A. M. Estudos antropológicos sobre os adoecidos crônicos. In: Olhares socioantropológicos sobre os adoecidos crônicos. São Paulo: Hucitec, 2007, p. 19-51.

CHAVES, J. H. B.; MENDONÇA, V. L. G.; PESSINI, L.; REGO, G.; NUNES, R. Cuidados paliativos na prática médica: contexto bioético. Rev Dor. São Paulo, 2011, v. 12, n. 3, p. 250-255.

DELORY-MOMBERGER, C. La condition biographique. Essais sur le récit de soi dans la modernité avancée. Paris : Téraèdre, coll. "Autobiographie et éducation ", 2010.

. Abordagens metodológicas na pesquisa biográfica. Revista Brasileira de Educação, 2012, v. 17, n. 51, p. 523-536.

. De la recherche biographique en éducation. Fondements Méthodes Pratiques. Paris : Téraèdre, coll. " Autobiographie et éducation ", 2014.

GUATTARI F. O novo paradigma estético. In: Caosmose: um novo paradigma estético. São Paulo: Editora 34. 1992, p. 125-148.

GUERINI, I. C.; CORDEIRO, P. K. S.; OSTA, S. Z.; RIBEIRO, E. M. Percepção de familiares sobre estressores decorrentes das demandas de cuidado de criança e adolescente dependentes de tecnologias. Texto Contexto Enferm, 2012, v. 21, n. 2, p. 348-55.

JOSSO, C. A transformação de si a partir da narração de histórias de vida. Educação, 2007 , v. 3, n. 63 , p. $413-438$.

KÜBLER-ROSS, E. La mort, dernière étape de la croissance. Paris: Edtions du Rocher, 1985.

LAPLANTINE F. Aprender antropologia. São Paulo: Brasiliense, 2003. 
MENEZES, R. A. Tecnologia e "Morte Natural": o Morrer na Contemporaneidade. PHYSIS: Rev. Saúde Coletiva, 2003, v. 13, n. 2, p. 129-147.

MENEZES, R. A.; BARBOSA, P. C. A construção da "boa morte" em diferentes etapas da vida: reflexões em torno do ideário paliativista para adultos e crianças. Ciência \& Saúde Coletiva, 2013 , v. 18 , n. 9 , p. 2653-2662.

MORITZ RD, DEICAS A, CAPALBO M, FORTE DN, KRETZER LP, LAGO P et al. II Fórum do "Grupo de Estudos do Fim da Vida do Cone Sul": definições, recomendações e ações integradas para cuidados paliativos na unidade de terapia intensiva de adultos e pediátrica. Rev Bras Ter Intensiva, 2011, v. 23, n. 1, p. 24-29.

ORGANIZAÇÃO MUNDIAL DA SAÚDE. Cuidados inovadores para condições crônicas: componentes estruturais de ação: relatório mundial - Brasília, 2003.

PINEAU G.; MARIE-MICHĖLE. Produire sa vie: autoformation et autobiographie, Montreal : Éditions Saint-Martin ; Paris ; Edilig, 2012.

RABELLO, C. A. F. G.; RODRIGUES, P. H. A. Saúde da família e cuidados paliativos infantis: ouvindo os familiares de crianças dependentes de tecnologia. Ciência e Saúde Coletiva, 2010 , v. 15 , n. 2, p. 379-388.

REMEDI P.P., et al. Cuidados paliativos para adolescentes com câncer: uma revisão da literatura. Rev. Bras. Enferm., 2009, v. 62, n. 1, p. 107-12.

SILVA K. S.; KRUSE, M. H. L. Em defesa da sociedade: a invenção dos cuidados paliativos e os dispositivos de segurança. Texto Contexto Enferm, 2013, v. 22, n. 2, p. 517-25.

SOUZA, L. F.; MISKO, M. D.; SILVA, L.; POLES, K.; SANTOS, M. R.; BOUSSO, R. S. Morte digna da criança: percepção de enfermeiros de uma unidade de oncologia. Rev Esc Enferm USP 2013, v. 47, n. 1, p. 30-37.

VIEIRA M. A.; LIMA, R.A.G. Crianças e adolescentes com doença crônica: convivendo com mudanças. Revista Latino-Americana de Enfermagem. 2002, v. 10, n. 4, p. 552-60. 